\section{ORIGINAL RESEARCH}

\author{
F. Rodjan \\ P. de Graaf \\ A.C. Moll \\ S.M. Imhof \\ J.I.M.L Verbeke \\ E. Sanchez
}

J.A. Castelijns

\title{
Brain Abnormalities on MR Imaging in Patients with Retinoblastoma
}

BACKGROUND AND PURPOSE: Although pineoblastoma is the main brain abnormality associated with hereditary retinoblastoma, recent studies suggest an association with pineal cysts. This association is important because some pineoblastomas mimic pineal cysts. If there is a relationship, then radiologists should be aware of it because diagnostic confusion is possible. Mental retardation and congenital brain anomalies are also reported in patients with retinoblastoma, mostly in combination with $13 q$ deletion syndrome. In this retrospective study, the presence of brain abnormalities on MR images in a large group of consecutive patients with retinoblastoma is evaluated.

MATERIALS AND METHODS: Brain MR images of 168 patients with retinoblastoma from 1989 to 2009 were evaluated by 2 radiologists for tumors, structural anomalies, myelinization, and coincidental findings. Clinical records were reviewed for laterality, heredity, and the presence of the $13 q$ deletion syndrome.

RESULTS: The hereditary group (patients with bilateral and unilateral proved RB1-germline mutation) included $90(54 \%)$ of 168 patients. Seven patients had 13q deletion syndrome. Normal findings on brain MR images were seen in 150 (89\%) patients. Five pineoblastomas were detected, all in patients with hereditary retinoblastoma $(5.5 \%$ in the hereditary subgroup). Nine pineal cysts were detected (2.2\% in the hereditary subgroup). Corpus callosum agenesis was found in 1 patient and a DandyWalker variant in 1 patient, both in combination with $13 q$ deletion syndrome.

CONCLUSIONS: Pineoblastoma is associated with hereditary retinoblastoma, and structural brain abnormalities are restricted to patients with the $13 q$ deletion syndrome. The incidence of pineal cysts in patients with retinoblastomas is similar to that in healthy children and is not associated with hereditary retinoblastoma.

ABBREVIATIONS: $\mathrm{CCA}=$ corpus callosum agenesis; $\mathrm{DV}=$ dilated ventricles; $\mathrm{MRI}=\mathrm{MR}$ imaging; Pre-t. $\mathrm{MRI}=$ pretreatment $\mathrm{MRI} ; \mathrm{RB}=$ retinoblastoma

$\mathbf{R}$ etinoblastoma is the most common intraocular tumor in early childhood, occurring in 1/17,000 live births. ${ }^{1}$ Retinoblastoma cells contain a mutation or deletion of the retinoblastoma gene ( $R B 1$ gene), a tumor-suppressor gene, located on chromosome $13 \mathrm{q} 14 .^{2,3}$ Approximately $40 \%$ of patients with retinoblastoma have hereditary disease (mostly bilateral tumors), while the remainder $(60 \%)$ have unilateral nonhereditary disease. A minority is due to a cytogenetically detectable interstitial deletion of variable size with a minimal overlapping region in band $13 \mathrm{q} 14 .{ }^{4}$ It is reported that midline intracranial neuroblastic tumors of either the suprasellar or the pineal region (pineoblastoma) are present in approximately $5 \%-15 \%$ of patients with hereditary retinoblastoma. ${ }^{5}$

Besides midline malignant tumors, benign intracranial abnormalities are also reported in the retinoblastoma population, in particular the presence of pineal cysts. In the literature, an association between hereditary retinoblastoma and pineal

Received December 23, 2009; accepted after revision February 17, 2010.

From the Departments of Radiology (F.R., P.d.G., J.I.M.L.V., E.S., J.A.C.) and Ophthalmology (A.C.M., S.M.I.) VU University Medical Center, Amsterdam, the Netherlands.

F.R. is financially supported by grants from the ODAS Foundation, Delft, the Netherlands; the National Foundation for the Blind and Visually Impaired, Utrecht, the Netherlands; and the Blindenhulp Foundation, 's-Gravenhage, the Netherlands.

Please address correspondence to Firazia Rodjan, MD, VU University Medical Center, Department of Radiology, Postbox 7057, 1007 MB Amsterdam, the Netherlands; e-mail: f.rodjan@vumc.nl

Indicates open access to non-subscribers at www.ajnr.org

DOI 10.3174/ajnr.A2102 cysts is suggested. Popovic et $\mathrm{al}^{6}$ reported an incidence of pineal lesions (cysts and pineoblastoma) of $6.4 \%$ in their retinoblastoma population. All pineal lesions were present in the bilaterally affected hereditary patients. In this subgroup, they found an equal incidence of $5.3 \%$ for both pineal cysts and pineoblastoma. The combination of retinoblastoma, suprasellar tumor, and pineal cystis is also reported, which, according to Popovic et $\mathrm{al}^{7}{ }^{7}$ further adds to a possible relationship between hereditary retinoblastoma and pineal lesions. Karatza et $\mathrm{al}^{8}$ reported 11 patients with retinoblastoma with pineal cysts simulating pineoblastoma. They recommended that clinicians should be aware of the presence of this benign lesion when performing routine neuroimaging, especially in patients with bilateral disease.

However, pineal cysts are quite common in the general population. In postmortem cases, pineal cysts are encountered in $25 \%-40 \%$ of people of all ages. ${ }^{9,10}$ In recent literature, an incidence of $0.4 \%-2.2 \%$ on MR images in the general pediatric population between 0 and 5 years of age has been reported. ${ }^{11}$ It is already known that pineoblastoma is associated with retinoblastoma. If the same is true for pineal cysts, then radiologists should be aware of the association. Distinct imaging criteria in distinguishing pineoblastomas from pineal cysts should be evaluated to avoid diagnostic and therapeutic confusion.

Retinoblastoma may occur in the presence of $13 \mathrm{q}$ deletion syndrome. The risk of retinoblastoma development in patients with this syndrome is approximately $80 \% .^{3,4}$ Besides retinoblastoma, this syndrome has a spectrum of clinical features, 
including moderate-to-severe developmental delay. Baud et $\mathrm{al}^{4}$ reported a variable degree of mental retardation in patients with retinoblastoma with associated $13 \mathrm{q}$ cytogenic abnormalities. Only in case reports, describing patients with $13 \mathrm{q}$ deletion syndrome or in combination with retinoblastoma, are congenital brain abnormalities or brain disorders indica-

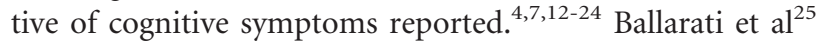
reported central nervous system anomalies such as DandyWalker malformation, cerebellar hypoplasia, cortical dysplasia, and agenesis of the corpus callosum in patients with $13 \mathrm{q}$ deletion. Although one can assume that these symptoms are due to $13 \mathrm{q}$ deletion syndrome, it is interesting to evaluate whether (structural) brain abnormalities are involved in patients with retinoblastoma. To our knowledge, no large correlative study has been performed on the presence of retinoblastoma and brain abnormalities on MR imaging.

The purpose of this retrospective study was to evaluate the presence of brain abnormalities on MR images in a large group of consecutive patients with retinoblastoma.

\section{Materials and Methods}

\section{Patients}

Patients eligible for this retrospective study were those at our institution with retinoblastoma who had undergone MR imaging of the brain that enabled adequate image interpretation between 1989 and 2009. To avoid alteration in the brain due to aging, we only evaluated patients younger than 5 years of age. The diagnosis of retinoblastoma was confirmed in all patients with extensive funduscopy, sonography, and MR imaging. In case of enucleation, the diagnosis was confirmed by histopathology. In this period, we identified 245 patients with retinoblastoma, and all patients had undergone MR imaging. However, in 77 patients, brain imaging studies were absent or incomplete and, therefore, excluded. The total number of included patients was 168 . The need for informed consent was waived because of the retrospective nature of the study.

Clinical records were reviewed by 1 reviewer for the patient's sex, age at first MR imaging, laterality, heredity, and the presence of $13 \mathrm{q}$ deletion syndrome. In patients with brain abnormalities, the evaluation of pre- or posttreatment MR imaging had to exclude treatmentinduced brain abnormalities.

Patients with bilateral disease or a positive family history of retinoblastoma or a defect in the $R B 1$ gene found in chromosomal/deoxyribonucleic acid analysis were classified as having hereditary disease. All other patients were classified as having nonhereditary disease. $^{1}$

\section{MR Imaging}

MR imaging was performed at 1T (Magnetom Impact Expert; Siemens, Erlangen, Germany) and 1.5T (Magnetom Vision and Sonata, Siemens). Within the past 20 years, MR imaging protocols for retinoblastoma have changed. In the beginning, whole-brain MR imaging sequences were more common in retinoblastoma MR imaging protocols in combination with a standard-quadrature head coil. Due to technologic developments and improved coil design, attention shifted to small-FOV imaging of the orbits by using surface coils. MR imaging protocols did not differ between unilaterally and bilaterally affected patients. Postcontrast axial T1-weighted MR images covering the whole brain were available in all included patients. In only 48 of 168 patients were axial T2-weighted MR images of the brain available.

\section{Image Analysis}

MR imaging examinations were individually reviewed by 2 radiologists with, respectively, 4 and 9 years of experience in pediatric neuroradiology. Disagreement was resolved by consensus. The parameters evaluated on MR imaging were selected on the basis of literature study ${ }^{4,7,12-24}$ and were divided into the following categories: benign or malignant tumor (tumor of the pineal gland, pineal cyst, or other); structural anomalies (corpus callosum agenesis, holoprosencephaly, encephalocele, Dandy-Walker malformation or a variant, hypoplasia of the basic pontis, microcephaly and polymicrogyria, or other); and degree of myelinization, ventriculomegaly, or coincidental findings. Special attention was paid to myelinization to clarify possible cognitive symptoms. The diagnosis of a pineal tumor was made if the pineal gland was enlarged, solid, and isointense to gray matter on T1weighted images with homogeneous and intense contrast enhancement. ${ }^{6}$ A pineal cyst was diagnosed if the pineal gland was enlarged and had a central region hypointense with respect to white matter on T1-weighted images and isointense with respect to CSF on T2weighted images and a thin wall of $\leq 2 \mathrm{~mm}$ with discrete rim enhancement after gadolinium injection. ${ }^{6}$ The diameters of pineal cysts and pineoblastomas were measured.

\section{Results}

\section{Clinical Findings}

The mean age at first MR imaging of the 168 patients was 17 months (range, 0-59 months). Bilateral retinoblastoma was present in 78 patients $(46 \%)$ and unilateral disease in 90 patients $(54 \%)$. Seventy-nine patients with retinoblastoma were female $(47 \%)$ (41 bilateral, 38 unilateral) and 89 (53\%) were male (36 bilateral, 53 unilateral). The hereditary group consisted of 90 patients and the nonhereditary group of 78 patients. Seven hereditary patients $(8 \%)$ were diagnosed with $13 \mathrm{q}$ deletion syndrome.

\section{MR Imaging Findings}

Brain MR imaging showed no abnormalities in 150 (89\%) patients. Brain abnormalities were found in 18 patients (11\%), with the pineal gland as the most commonly affected site (14 patients, 78\%) (Table).

Pineoblastoma was found in 5 patients at a mean age of 20 months (range, 1-39 months), all with hereditary retinoblastoma $(3.0 \%$ overall incidence, and $5.5 \%$ in the hereditary group). The mean interval between diagnosis of ocular and intracranial tumor was 16.4 months. In 1 patient (10 months of age), the pineoblastoma was detected with the diagnosis of retinoblastoma (synchronous tumors). In the other 4 patients, the pineoblastoma was discovered at a later stage; $6,15,25$, and 37 months after the diagnosis of retinoblastoma (metachronous tumors). In patients 6,8 , and 9 , the tumor presented as a solid mass isointense with respect to gray matter on precontrast T1-weighted images and showed a homogeneous intense contrast enhancement in the pineal region (diameters respectively, 55, 16, and $49 \mathrm{~mm}$ ) (Table). In patients 5 and 7 , the tumor consisted of a heterogeneous mass with intense contrast enhancement and areas with low signal intensity on T1weighted images and high signal intensity on T2-weighted images, suspicious for central necrosis. In patient 5, the configuration of the lesion mimicked a pineal cyst (Fig 1). On axial images however, the diagnosis of pineoblastoma was more 


\begin{tabular}{|c|c|c|c|c|}
\hline \multicolumn{5}{|c|}{ MR imaging findings in patients with retinoblastoma } \\
\hline $\begin{array}{l}\text { Patient No./ } \\
\text { Age (Mo)/Sex }\end{array}$ & Heredity & Pre-t. MRI & MRI Findings (Side) & Size $(\mathrm{mm})$ \\
\hline$\overline{1 / 1 / M}$ & Yes & Yes & DV (temporal and atrium) & \\
\hline 2/13/M & Yes & Yes & Dandy-Walker variant with DV & \\
\hline $3 / 4 / F$ & Yes & Yes & CCA (corpus/splenium), vermis hypoplasia, DV & \\
\hline 4/10/M & Yes & Yes & Trigonocephalia, DV & \\
\hline $5 / 10 / \mathrm{M}$ & Yes & Yes & Pineoblastoma & 15 \\
\hline $6 / 39 / F$ & Yes & Yes & Pineoblastoma & 55 \\
\hline $7 / 5 / F$ & Yes & Yes & Pineoblastoma & 59 \\
\hline $8 / 1 / \mathrm{M}$ & Yes & Yes & Pineoblastoma & 16 \\
\hline $9 / 37 / F$ & Yes & Yes & Pineoblastoma & 49 \\
\hline $10 / 34 / \mathrm{M}$ & No & Yes & Pineal cyst & 7 \\
\hline $11 / 16 / F$ & No & Yes & Pineal cyst & 6 \\
\hline $12 / 31 / \mathrm{M}$ & No & Yes & Pineal cyst & 6 \\
\hline $13 / 15 / F$ & Yes & Yes & Pineal cyst & 5 \\
\hline $14 / 10 / F$ & No & Yes & Pineal cyst & 4 \\
\hline $15 / 23 / F$ & No & Yes & Pineal cyst & 3 \\
\hline $16 / 9 / F$ & Yes & Yes & Pineal cyst & 3 \\
\hline 17/10/M & No & Yes & Pineal cyst & 4 \\
\hline 18/18/M & No & Yes & Pineal cyst & 3 \\
\hline
\end{tabular}
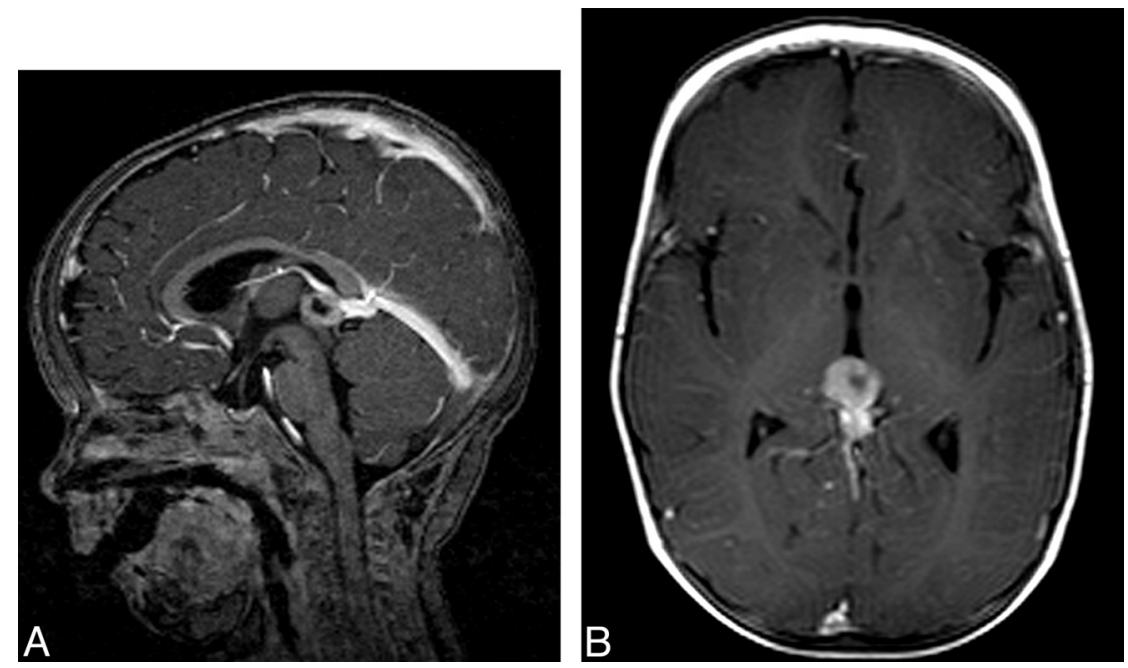

Fig 1. Patient 5, a 10-month-old with hereditary retinoblastoma and pineoblastoma. A, Sagittal T1-weighted postgadolinium MR image shows a cystic mass in the pineal gland. $B$, Axial T1-weighted image shows an asymmetric nodular thickening of the tumor wall. The thickness of the enhancing wall varies from 3 to $7 \mathrm{~mm}$, suspicious for pineoblastoma.

evident because there was an asymmetric nodular thickening of the enhancing wall. The thickness of the enhancing wall varied from 3 to $7 \mathrm{~mm}$. Histopathology revealed a pineoblastoma with central necrosis.

The total incidence of pineal cysts in our study was 5.4\% ( 9 patients), with an incidence of $9.0 \%$ in the nonhereditary group (7 patients; mean age, 20 months; range, 10-34 months) and $2.2 \%$ in the hereditary group (2 patients; mean age, 9 months; range, 9-10 months) (Fig 2). The mean size of the cysts in the nonhereditary group was $4.8 \mathrm{~mm}$ (range, 3-7 $\mathrm{mm}$ ) and $4 \mathrm{~mm}$ in the hereditary group (range, 3-5 $\mathrm{mm}$ ). In 2 patients, follow-up MR imaging was available and showed no change in the pineal cysts.

Besides pineoblastoma and pineal cysts, a diversity of brain abnormalities was found. Corpus callosum agenesis occurred in 1 patient with hereditary retinoblastoma (Fig 3), and in another patient, a hereditary Dandy-Walker variant occurred. Both were known to have a $13 \mathrm{q}$ deletion syndrome. In the other 5 patients (71\%) with 13q deletion syndrome, no brain abnormalities were present. One patient with hereditary retinoblastoma showed DV.
Evaluation of white matter abnormalities was only possible in 48 patients because of incomplete series of T2-weighted images of the brain in 120 patients. In this subgroup, no white matter abnormalities were observed and all patients with retinoblastoma showed normal myelinization.

\section{Discussion}

In this study, we present an overview of brain abnormalities in a large group of patients with retinoblastoma.

Pineoblastoma is the most well-known brain pathology associated with retinoblastoma, a combination known as trilateral retinoblastoma, occurring in $1.5 \%-5 \%$ of all patients and in $5 \%-15 \%$ in the hereditary subgroup. ${ }^{5,22}$ Besides the pineal region, tumors may also occur in the suprasellar or parasellar region. Our study showed similar results, with an incidence of $3.0 \%$ in the whole study group and 5.5\% concerning only the hereditary group.

Three of 5 pineoblastomas showed a typical imaging pattern: a solid homogeneous lobulated enlarged pineal gland, isointense with gray matter on T1-weighted images with intense homogeneous contrast-enhancement. In the other 2 


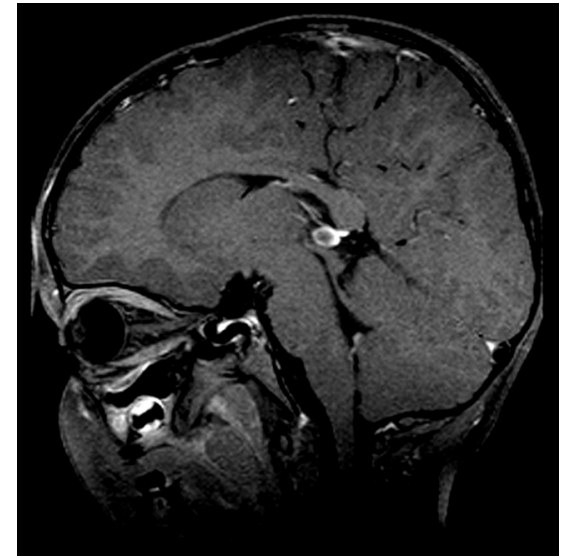

Fig 2. Patient 10. Contrast-enhanced T1-weighted MR image of a 34-month-old patient with nonhereditary retinoblastoma with a pineal cyst, with a diameter of $7 \mathrm{~mm}$.

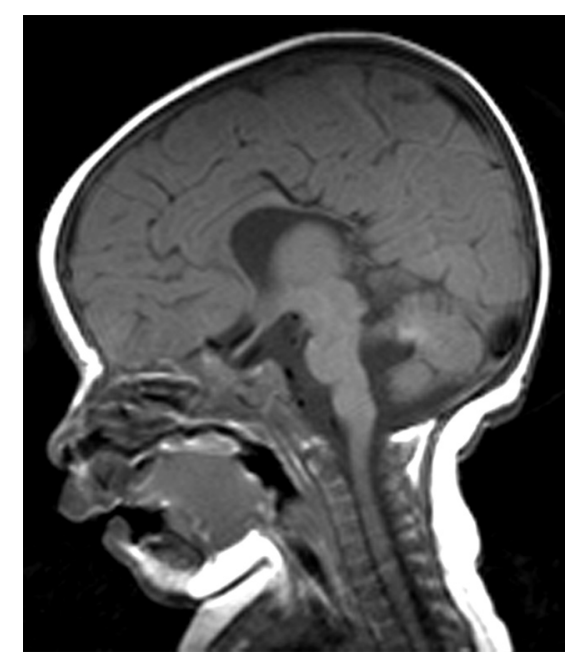

Fig 3. Patient 3 . Sagittal T1-weighted image of a 4-month-old with $13 q$ deletion syndrome and bilateral retinoblastoma shows agenesis of the splenium of the corpus callosum.

pineoblastomas, low signal intensity on T1-weighted images and high signal intensity on T2-weighted images with heterogeneous enhancement areas were present. One pineoblastoma even mimicked a pineal cyst due to a large central area of histopathologically proved necrosis. The thickness of this cyst wall $(7 \mathrm{~mm})$, however, did not correspond with the criteria of pineal cyst (thickness, $\leq 2 \mathrm{~mm}$ ). This finding can cause confusion in the diagnosis of pineoblastoma, and it could have been erroneously diagnosed as a pineal cyst, with severe clinical consequences. ${ }^{8}$ This has been reported only once before. Sugiyama et $\mathrm{al}^{26}$ reported a case of a pineoblastoma with a large central cyst, histopathologically due to central necrosis. In our patient, the pineoblastoma was discovered in an early stage synchronously with the intraocular tumor on baseline MR imaging without clinical symptoms due to pineoblastoma.

Central necrosis can be present in large and small pineoblastomas, and especially small pineoblastomas with central necrosis can mimic a pineal cyst. In our experience, the thickness and appearance of the cyst wall before and after contrast administration on MR imaging are indeed important to differentiate a pineal cyst and pineoblastoma. Pineal cysts show a thin wall $(<2 \mathrm{~mm})$ with discrete enhancement, whereas pine- oblastomas with central necrosis show a nodular thickened wall with homogeneous wall enhancement.

In our screening for brain abnormalities in 168 patients with retinoblastoma, pineal cysts were detected in $5.4 \%$ of the patients. Sener ${ }^{10}$ performed a review of MR imaging in 500 children and 500 adults and found no pineal cysts in children younger than 12 years of age. The sensitivity of this study, however, could have been limited by using $0.5 \mathrm{~T}$ MR imaging in evaluation. Sawamura et $\mathrm{al}^{27}$ found 79 cysts in 6023 patients but none in children younger than 10 years of age. However, only MR images in 73 children of 10 years of age and younger were evaluated.

The study of Al-Holou et $\mathrm{al}^{11}$ was the only one in which the incidence of pineal cysts on MR imaging was examined in healthy children younger than 5 years of age. They reported an incidence of $2.0 \%$, almost similar to that in our hereditary group. In this study, the pineal cyst population was compared with an age- and sex-matched control population. Popovic et $\mathrm{al}^{6,7}$ also reported almost the same incidence as in our overall study group (5.3\%). They found cysts in patients with bilateral hereditary retinoblastoma, but no pineal cysts in the patients with nonhereditary retinoblastoma. These previously mentioned studies included only pineal cysts of $>5 \mathrm{~mm}$. In our study of patients with retinoblastoma younger than 5 years of age, 5 of the 9 cysts were $<5 \mathrm{~mm}$ ( 4 of the 5 pineal cysts in the nonhereditary group).

A follow-up study in a matched control group would be necessary to examine the high incidence of pineal cysts in the patients with nonhereditary compared with hereditary retinoblastoma with the same criteria used in our study. In previous studies, only Karatza et $\mathrm{al}^{8}$ reported the occurrence of pineal cysts in patients with nonhereditary retinoblastoma. They reviewed 1400 medical records of patients with retinoblastoma and found 3 pineal cysts $(0.2 \%)$ in patients with unilateral and 8 pineal cysts $(0.6 \%)$ in patients with bilateral retinoblastoma. These low incidences are probably due to screening for pineal cysts in medical records instead of re-evaluation of MR images. In our experience, these cysts are not always described in medical records as a finding. However, for assessing the association between retinoblastoma and pineal cysts, the incidence of pineal cysts in the hereditary group is interesting. Given the incidence of $2.2 \%$ in the hereditary group, which is similar to the incidence in the healthy population, ${ }^{11}$ there is no association between hereditary retinoblastoma and pineal cysts. Although it is important to be aware of pineoblastomas mimicking a pineal cyst, there is no evidence that pineal cysts occur more often in patients with retinoblastoma compared with the healthy pediatric population.

In our study group, larger structural brain abnormalities occurred only in combination with a 13 q deletion syndrome. One patient with $13 \mathrm{q}$ deletion syndrome had a corpus callosum agenesis and another patient showed a Dandy-Walker variant with DV. This brain abnormality is also described in previous case reports. Alanay et $\mathrm{al}^{28}$ reported a patient with a thin corpus callosum and a Dandy-Walker malformation.

\section{Conclusions}

In conclusion, pineoblastoma is associated with hereditary retinoblastoma, and structural brain abnormalities are restricted to the patients with $13 \mathrm{q}$ deletion syndrome. The inci- 
dence of pineal cysts in retinoblastoma is similar to that in healthy children and is not associated with hereditary retinoblastoma.

\section{References}

1. Moll AC, Kuik DJ, Bouter LM, et al. Incidence and survival of retinoblastoma in The Netherlands: a register based study 1862-1995. Br J Ophthalmol 1997;81:559-62

2. Friend SH, Bernards R, Rogelj S, et al. A human DNA segment with properties of the gene that predisposes to retinoblastoma and osteosarcoma. Nature 1986;323:643-46

3. Ganesh A, Kenue RK, Mitra S. Retinoblastoma and the 13q deletion syndrome. J Pediatr Ophthalmol Strabismus 2001;38:247-50

4. Baud O, Cormier-Daire V, Lyonnet S, et al. Dysmorphic phenotype and neurological impairment in 22 retinoblastoma patients with constitutional cytogenetic 13q deletion. Clin Genet 1999;55:478-82

5. Kivela $T$. Trilateral retinoblastoma: a meta-analysis of hereditary retinoblastoma associated with primary ectopic intracranial retinoblastoma. J Clin $\mathrm{On}$ col 1999;17:1829-37

6. Popovic MB, Balmer A, Maeder P, et al. Benign pineal cysts in children with bilateral retinoblastoma: a new variant of trilateral retinoblastoma? Pediatr Blood Cancer 2006;46:755-61

7. Popovic MB, Diezi M, Kuchler H, et al. Trilateral retinoblastoma with suprasellar tumor and associated pineal cyst. J Pediatr Hematol Oncol 2007;29:53-56

8. Karatza EC, Shields CL, Flanders AE, et al. Pineal cyst simulating pinealoblastoma in 11 children with retinoblastoma. Arch Ophthalmol 2006;124:595-97

9. Fleege MA, Miller GM, Fletcher GP, et al. Benign glial cysts of the pineal gland: unusual imaging characteristics with histologic correlation. AJNR Am J Neuroradiol 1994;15:161-66

10. Sener RN. The pineal gland: a comparative MR imaging study in children and adults with respect to normal anatomical variations and pineal cysts. Pediatr Radiol 1995;25:245-48

11. Al-Holou WN, Garton HJ, Muraszko KM, et al. Prevalence of pineal cysts in children and young adults: clinical article. J Neurosurg Pediatr 2009;4:230-36

12. Araujo JE, Filho HA, Pires CR, et al. Prenatal diagnosis of the 13q-syndrome through three-dimensional ultrasonography: a case report. Arch Gynecol $\mathrm{Ob}$ stet 2006;274:243-45

13. Chung JL, Choi JR, Park MS, et al. A case of $\operatorname{del}(13)(\mathbf{q} 2)$ with multiple major congenital anomalies, imperforate anus and penoscrotal transposition. Yonsei Med J 2001;42:558-62

14. Desai VN, Shields CL, Shields JA, et al. Retinoblastoma associated with holoprosencephaly. Am J Ophthalmol 1990;109:355-56

15. Duncan JL, Scott IU, Murray TG, et al. Routine neuroimaging in retinoblastoma for the detection of intracranial tumors. Arch Ophthalmol 2001;119:450-52

16. Ibarra MS, O'Brien JM. Is screening for primitive neuroectodermal tumors in patients with unilateral retinoblastoma necessary? J AAPOS 2000;4:54-56

17. Kasyan AG, Benirschke K. Genetic haploinsufficiency as a phenotypic determinant of a deletion 13q syndrome. Pediatr Dev Pathol 2005;8:658-65. Epub 2005 Nov 18

18. Kennerknecht I, Barbi G, Greher J. Diagnosis of retinoblastoma in a presymptomatic stage after detection of interstitial chromosomal deletion 13q. Ophthalmic Genet 1994;15:19-24

19. Koestenberger M, Kroisel PM, Lackner H, et al. Simultaneous occurrence of retinoblastoma and neurofibromatosis I in a young child. Med Pediatr Oncol 2003;40:124-25

20. Kogan JM, Egelhoff JC, Saal HM. Interstitial deletion of $13 q$ associated with polymicrogyria. Am J Med Genet A 2008;146:910-16

21. Melis D, Pia Sperandeo M, Perone L, et al. Mosaic 13q13.2-ter deletion restricted to tissues of ectodermal and mesodermal origins. Clin Dysmorphol 2006;15:13-18

22. Provenzale JM, Gururangan S, Klintworth G. Trilateral retinoblastoma: clinical and radiologic progression. AJR Am J Roentgenol 2004;183:505-11

23. Skrypnyk C, Bartsch O. Retinoblastoma, pinealoma, and mild overgrowth in a boy with a deletion of RB1 and neighbor genes on chromosome 13q14. Am J Med Genet A 2004;124A:397-401

24. Skulski M, Egelhoff JC, Kollias SS, et al. Trilateral retinoblastoma with suprasellar involvement. Neuroradiology 1997;39:41-43

25. Ballarati L, Rossi E, Bonati MT, et al. 13q deletion and central nervous system anomalies: further insights from karyotype-phenotype analyses of 14 patients. J Med Genet 2007;44:e60

26. Sugiyama K, Arita K, Okamura T, et al. Detection of a pineoblastoma with large central cyst in a young child. Childs Nerv Syst 2002;18:157-60

27. Sawamura Y, Ikeda J, Ozawa $M$, et al. Magnetic resonance images reveal a high incidence of asymptomatic pineal cysts in young women. Neurosurgery 1995;37:11-15

28. Alanay Y, Aktas D, Utine E, et al. Is Dandy-Walker malformation associated with "distal 13q deletion syndrome"? Findings in a fetus supporting previous observations. Am J Med Genet A 2005;136:265-68 